九州大学学術情報リポジトリ

Kyushu University Institutional Repository

\title{
ON EXPECTED VALUES OF MARKOV STATISTICS
}

Iwamoto, Seiichi

Department of Economic Engineering, Faculty of Economics, Kyushu University

https://doi.org/10.5109/13466

出版情報: Bulletin of informatics and cybernetics. 30 (1)，pp.1-24，1998-03. Research Association of Statistical Sciences

バージョン :

権利関係 : 


\title{
ON EXPECTED VALUES OF MARKOV STATISTICS
}

\author{
By
}

\author{
Seiichi Iwamoto*
}

\begin{abstract}
In this paper we give both forward and backward iterative algorithms for computing expected value of some associative statistics associated with a stationary Markov chain on finite state space. Both algorithms are based upon an invariant imbedding technique in dynamic programming.
\end{abstract}

\section{Introduction}

In this paper we consider how to compute the expected value of some associative statistics from a stationary Markov chain on a finite state space. We are concerned with two kinds of related associative statistics. One is simple statistics. The other is compound one. The simple statistics include sample sum, sample mean, sample maximum, sample minimum, and others from iid populations. As three compound ones we consider range statistics, ratio statistics, and variance statistics from the Markov chain (Sniedovich $(1983,1987,1989,1992)$ ).

Introducing a new real-parameter at the head of statistics we imbed the expectation problem into a family of parametric problems, one of which reduces to the original problem. We show both forward and backward iterative algorithms for computing the expected value. Both algorithms are based upon an invariant imbedding technique (Bellman and Denman(1971), Iwamoto(1996), Iwamoto and Fujita(1995), Lee(1968), Sniedovich $(1983,1989)$ ) in stochastic dynamic programming (Bellman(1957), Blaclwell (1965), Denardo(1968, 1972), Furukawa and Iwamoto(1973a, 1973b), Hinderer(1970), Howard(1960), Iwamoto(1974, 1975a, 1975b, 1977, 1993, 1994), Kreps(1977a, 1977b), Lipfert(1985), Mitten(1964), Nemhauser(1966), Porteus(1975, 1982), Puterman(1994), Sniedovich(1986)).

In Section 2, we define associative statistics from Markov chain. Deriving the conditional probability function, we give the direct computation method for associative statistics. Not only for associative statistics but also for functions of it we give two iterative computation methods, based upon forward and backward recursive equations in dynamic programming.

\footnotetext{
* Department of Economic Engineering, Faculty of Economica, Kyushu University 27, Fukuoka 812-81, Japan

e-mail: iwamoto@en.kyushu-u.ac.jp
} 
In Section 3, as two examples of associative statistics, we consider extremum statistics from Markov chain: maximum statistics and minimum statistics. We specify forward and backward iterative algorithms. In Section 4, as three examples of compound statistics, we consider range statistics, ratio statistics, and variance statistics from Markov chain. Imbedding the original problem into an appropriately large family of parametric ones, we derive forward and backward iterative algorithms. In Section 5, we illustrate typical examples both of associative binary relation and of nonassociative one. In Section 6, we give a numeical example, which asșures the same expected value through three methods: direct computation, forward iterative computation, and backward computation. In the last section we conclude with some remarks on associative/nonassociative statistics and stationary/nonstationary and forward/backward recursive equations.

\section{Associative Statistics and Related Statistics}

Let $S=\{1,2, \ldots, N\}$ be a finite state space, where $N \geq 1$ is an integer. Throughout the paper, let $X_{n}, n \geq 0$ be a stationary Markov chain on $S$ having the one-step transition function $p(j \mid i)$ :

$$
p(j \mid i)=P\left(X_{n+1}=j \mid X_{n}=i\right) \quad i \in S, \quad j \in S \quad n \geq 0 .
$$

It is such that

and

$$
p(j \mid i) \geq 0 \quad i \in S, \quad j \in S,
$$

$$
\sum_{j \in S} p(j \mid i)=1 \quad i \in S .
$$

It now follows from the stationary Markov property that

$$
\begin{aligned}
& P\left(X_{m+n+1}=j, X_{m+n+2}=k, \cdots, X_{m+n+l-1}=s, X_{m+n+l}=t\right. \\
&\left.\mid X_{m+n}=i, X_{m+n-1}=i_{n-1}, \cdots, X_{m+1}=i_{1}, X_{m}=i_{0}\right) \\
&= P\left(X_{m+n+1}=j, X_{m+n+2}=k, \cdots, X_{m+n+l-1}=s, X_{m+n+l}=t \mid X_{m+n}=i\right) \\
&= p(t \mid s) \cdots p(k \mid j) p(j \mid i) .
\end{aligned}
$$

We also remark that

$$
\begin{aligned}
& P\left(X_{1}=j, X_{2}=k, \cdots, X_{n-1}=s, X_{n}=t \mid X_{1}=j, X_{0}=i\right) \\
= & P\left(X_{2}=k, X_{3}=l, \cdots, X_{n-1}=s, X_{n}=t \mid X_{1}=j\right) \\
= & p(t \mid s) \cdots p(l \mid k) p(k \mid j) .
\end{aligned}
$$

Let $R \subset R^{1}$ be any interval. Let $\circ: R \times R \rightarrow R$ be an associative binary relation on $R$ :

Any $\tilde{x}$ satisfying

$$
(x \circ y) \circ z=x \circ(y \circ z)
$$

$$
\tilde{x} \circ y=y \quad \forall y \in R
$$

is called a left-identity element for $\circ$. The common value (1) is denoted by $x \circ y \circ z$. We also use the notation $x_{1} \circ x_{2} \circ \cdots \circ x_{n}$ in the following. 


\subsection{Associative Statistics}

Let $r: S \rightarrow R$ be a function. We consider how to compute the conditional expected value of the related associative statistics $R_{0} \circ R_{1} \circ \cdots \circ R_{n}$ :

$$
u_{n}(i)=E\left[R_{0} \circ R_{1} \circ \cdots \circ R_{n} \mid X_{0}=i\right] \quad i \in S
$$

where

$$
R_{k}=r\left(X_{k}\right)
$$

First, we consider the direct computation as follows. The conditional probability distribution $p_{n}\left(x_{0}, x_{1}, x_{2}, \cdots, x_{n-1}, x_{n} \mid x_{0}\right)$ of $\left(X_{0}, X_{1}, X_{2}, \cdots, X_{n-1}, X_{n}\right)$ given $X_{0}=i$ becomes

$$
\begin{aligned}
& p_{n}(j, k, l, \cdots, s, t \mid i) \\
= & P\left(X_{0}=j, X_{1}=k, X_{2}=l, \cdots, X_{n-1}=s, X_{n}=t \mid X_{0}=i\right) \\
= & p(t \mid s) \cdots p(l \mid k) p(k \mid j) \delta_{i j}
\end{aligned}
$$

where $\delta_{i j}$ is the Dirac's notation :

$$
\delta_{i j}= \begin{cases}1 & \text { for } i=j \\ 0 & \text { for } i \neq j\end{cases}
$$

Then we have

$$
\begin{aligned}
u_{n}(i) & =\sum_{j, k, \ldots, s, t}[r(j) \circ r(k) \circ r(l) \circ \cdots \circ r(s) \circ r(t)] p_{n}(j, k, l, \cdots, s, t \mid i) \\
& =\sum_{j, k, \ldots, s, t}[r(j) \circ r(k) \circ r(l) \circ \cdots \circ r(s) \circ r(t)] p(t \mid s) \cdots p(l \mid k) p(k \mid j) \delta_{i j} \\
& =\sum_{k, \ldots, s, t}[r(i) \circ r(k) \circ r(l) \circ \cdots \circ r(s) \circ r(t)] p(t \mid s) \cdots p(l \mid k) p(k \mid i)
\end{aligned}
$$

where each of $j, k, \ldots, s, t(k, \ldots, s, t)$ ranges over $S=\{1,2, \ldots, N\}$.

Second, we imbed the problem (2) into the class of parametrized problems:

$$
u_{n}(i ; \lambda)=E\left[\lambda \circ R_{0} \circ R_{1} \circ \cdots \circ R_{n} \mid X_{0}=i\right] \quad \lambda \in R .
$$

Then the stationarity implies that

$$
u_{n}(i ; \lambda)=E\left[\lambda \circ R_{k} \circ R_{k+1} \circ \cdots \circ R_{k+n} \mid X_{k}=i\right] \quad k \geq 0 .
$$

We have for any left-identity value $\tilde{\lambda}$

$$
u_{n}(i ; \tilde{\lambda})=u_{n}(i)
$$

The Markov property together with the associativity in $\circ$ yields

$$
\begin{aligned}
& E\left[\lambda \circ R_{0} \circ R_{1} \circ \cdots \circ R_{n} \mid X_{0}=i\right] \\
= & E\left[E\left[\left(\lambda \circ R_{0}\right) \circ R_{1} \circ \cdots \circ R_{n} \mid X_{1}, X_{0}=i\right] \mid X_{0}=i\right] \\
= & E\left[E\left[(\lambda \circ r(i)) \circ R_{1} \circ \cdots \circ R_{n} \mid X_{1}\right] \mid X_{0}=i\right] .
\end{aligned}
$$


Thus, we get the forward recurrence equation:

$$
\begin{aligned}
& u_{0}(i ; \lambda)=\lambda \circ r(i) \\
& u_{n}(i ; \lambda)=\sum_{j=1}^{N} u_{n-1}(j ; \lambda \circ r(i)) p(j \mid i) \quad n \geq 1
\end{aligned}
$$

Moreover, let $\pi_{0}=\left\{\pi_{0}(i)\right\}_{i \in S}$ be an initial distribution:

$$
P\left(X_{0}=i\right)=\pi_{0}(i) \geq 0 \quad i \in S
$$

and

$$
\sum_{i \in S} \pi_{0}(i)=1
$$

Then the (unconditional) expected value of the associative statistics $R_{0} \circ R_{1} \circ \cdots \circ R_{n}$ :

$$
u_{n}=E\left[R_{0} \circ R_{1} \circ \cdots \circ R_{n}\right] \quad n \geq 1
$$

is calculated as follows:

$$
u_{n}=\sum_{i=1}^{N} \pi_{0}(i) u_{n}(i) .
$$

On the other hand, we imbed the problem (2) into the class of backward problems:

$$
u^{n-k+1}(i ; \lambda)=E\left[\lambda \circ R_{k} \circ R_{k+1} \circ \cdots \circ R_{n} \mid X_{k}=i\right] \quad 0 \leq k \leq n, \quad \lambda \in R^{1} .
$$

We have for any left-identity value $\tilde{\lambda}$

$$
u^{n+1}(i ; \tilde{\lambda})=u_{n}(i)
$$

and the backward recurrence equation:

$$
\begin{aligned}
u^{1}(i ; \lambda) & =\lambda \circ r(i) \\
u^{n-k+1}(i ; \lambda) & =\sum_{j=1}^{N} u^{n-k}(j ; \lambda \circ r(i)) p(j \mid i) \quad 0 \leq k \leq n-1 .
\end{aligned}
$$

We remark that the stationarity implies the relation between forward and backward problems (3), (6):

$$
u^{n-k+1}(i ; \lambda)=u_{n-k}(i ; \lambda)
$$

\subsection{Function of Associative Statistics}

Let $h: R \rightarrow R^{1}$ be a function. Then we consider the conditional expected value of the function of $R_{0} \circ R_{1} \circ \cdots \circ R_{n}$ :

$$
v_{n}(i)=E\left[h\left(R_{0} \circ R_{1} \circ \cdots \circ R_{n}\right) \mid X_{0}=i\right] \quad i \in S
$$


First, we imbed this problem into the class of parametrized problems:

$$
v_{n}(i ; \lambda)=E\left[h\left(\lambda \circ R_{0} \circ R_{1} \circ \cdots \circ R_{n}\right) \mid X_{0}=i\right] \quad \lambda \in R
$$

We get the forward recurrence equation:

$$
\begin{aligned}
& v_{0}(i ; \lambda)=h(\lambda \circ r(i)) \\
& v_{n}(i ; \lambda)=\sum_{j=1}^{N} v_{n-1}(j ; \lambda \circ r(i)) p(j \mid i) \quad n \geq 1
\end{aligned}
$$

On the other hand, we imbed the problem (9) into the class of backward problems:

$$
v^{n-k+1}(i ; \lambda)=E\left[h\left(\lambda \circ R_{k} \circ R_{k+1} \circ \cdots \circ R_{n}\right) \mid X_{k}=i\right] \quad 0 \leq k \leq n, \quad \lambda \in R .
$$

We have for any left-identity $\tilde{\lambda}$

$$
v^{n+1}(i ; \tilde{\lambda})=v_{n}(i)
$$

and the backward recurrence equation:

$$
\begin{aligned}
v^{1}(i ; \lambda) & =h(\lambda \circ r(i)) \\
v^{n-k+1}(i ; \lambda) & =\sum_{j=1}^{N} v^{n-k}(j ; \lambda \circ r(i)) p(j \mid i) \quad 0 \leq k \leq n-1
\end{aligned}
$$

The stationarity also yields

$$
v^{n-k+1}(i ; \lambda)=v_{n-k}(i ; \lambda)
$$

\section{Single Statistics}

Let $f: S \rightarrow R^{1}$ and $g: S \rightarrow R^{1}$ be two functions. Then we define

$$
\begin{array}{cl}
Y_{n}=f\left(X_{0}\right) \vee f\left(X_{1}\right) \vee \cdots \vee f\left(X_{n}\right) & n \geq 0 \\
Z_{n}=g\left(X_{0}\right) \wedge g\left(X_{1}\right) \wedge \cdots \wedge g\left(X_{n}\right) & n \geq 0 \\
Y_{k, n}=f\left(X_{k}\right) \vee f\left(X_{k+1}\right) \vee \cdots \vee f\left(X_{n}\right) & 0 \leq k \leq n \\
Z_{k, n}=g\left(X_{k}\right) \wedge g\left(X_{k+1}\right) \wedge \cdots \wedge g\left(X_{n}\right) & 0 \leq k \leq n
\end{array}
$$

and

$$
m=\min \{f(x) \mid x \in S\} \quad M=\max \{f(x) \mid x \in S\}
$$




\subsection{Maximum Statistics}

First, we consider the conditional expected value :

$$
u_{n}(i)=E\left[Y_{n} \mid X_{0}=i\right] \quad n \geq 0 .
$$

We imbed this problem into the class of parametrized problems:

$$
u_{n}(i ; \lambda)=E\left[\lambda \vee Y_{n} \mid X_{0}=i\right] \quad m \leq \lambda \leq M .
$$

Then for a sufficiently small value $\tilde{\lambda}$ of $\lambda$ we have

$$
u_{n}(i ; \tilde{\lambda})=u_{n}(i)
$$

In particular,

$$
u_{n}(i ; m)=u_{n}(i) .
$$

We get the forward recurrence equation:

$$
\begin{aligned}
& u_{0}(i ; \lambda)=\lambda \vee f(i) \\
& u_{n}(i ; \lambda)=\sum_{j=1}^{N} u_{n-1}(j ; \lambda \vee f(i)) p(j \mid i) \quad n \geq 1 .
\end{aligned}
$$

On the other hand, we imbed the problem (10) into the class of backward problems:

$$
u^{n-k+1}(i ; \lambda)=E\left[\lambda \vee Y_{k, n} \mid X_{k}=i\right] \quad 0 \leq k \leq n, \quad m \leq \lambda \leq M .
$$

We have

$$
u^{n+1}(i ; m)=u_{n}(i)
$$

and the backward recurrence equation:

$$
\begin{aligned}
u^{1}(i ; \lambda) & =\lambda \vee f(i) \\
u^{n-k+1}(i ; \lambda) & =\sum_{j=1}^{N} u^{n-k}(j ; \lambda \vee f(i)) p(j \mid i) \quad 0 \leq k \leq n-1 .
\end{aligned}
$$

\subsection{Minimum Statistics}

Second, we consider the conditional expected value :

$$
v_{n}(i)=E\left[Z_{n} \mid X_{0}=i\right] \quad n \geq 0 .
$$

(See also Bellman and Zadeh(1970), Esogbue and Bellman(1984), Iwamoto(1996), Iwamoto and Fujita(1965), Kacprzyk(1978) for the corresponding optimization problem). We imbed this problem into the class of parametrized problems:

$$
v_{n}(i ; \mu)=E\left[\mu \wedge Z_{n} \mid X_{0}=i\right] \quad m \leq \mu \leq M .
$$


Then for a sufficiently large value $\tilde{\mu}$ of $\mu$ we have

$$
v_{n}(i ; \tilde{\mu})=v_{n}(i)
$$

In particular,

$$
v_{n}(i ; M)=v_{n}(i)
$$

We get the recurrence equation:

$$
\begin{aligned}
& \dot{v}_{0}(i ; \mu)=\mu \wedge f(i) \\
& v_{n}(i ; \mu)=\sum_{j=1}^{N} v_{n-1}(j ; \mu \wedge f(i)) p(j \mid i) \quad n \geq 1 .
\end{aligned}
$$

On the other hand, we imbed the problem (11) into the class of backward problems:

$$
v^{n-k+1}(i ; \mu)=E\left[\mu \wedge Z_{k, n} \mid X_{k}=i\right] \quad 0 \leq k \leq n, \quad m \leq \mu \leq M .
$$

We have

$$
v^{n+1}(i ; M)=v_{n}(i)
$$

and the backward recurrence equation:

$$
\begin{aligned}
v^{1}(i ; \mu) & =\mu \wedge f(i) \\
v^{n-k+1}(i ; \mu) & =\sum_{j=1}^{N} v^{n-k}(j ; \mu \wedge f(i)) p(j \mid i) \quad 0 \leq k \leq n-1
\end{aligned}
$$

\section{Compound Statistics}

In this section we consider three kinds of compound statistics; range, ratio and variance.

\subsection{Range Statistics}

Let $h: R^{1} \times R^{1} \rightarrow R^{1}$ be a function. First, we consider the conditional expected value :

$$
u_{n}(i)=E\left[h\left(Y_{n}, Z_{n}\right) \mid X_{0}=i\right] \quad n \geq 0
$$

If

$$
h(x, y)=x-y,
$$

then $u_{n}(i)$ represents a range for the statistics $X_{0}, X_{1}, \cdots, X_{n}$ :

$$
u_{n}(i)=E\left[Y_{n}-Z_{n} \mid X_{0}=i\right] \quad n \geq 0 .
$$

We have

$$
E\left[Y_{n}-Z_{n} \mid X_{0}=i\right]=E\left[Y_{n} \mid X_{0}=i\right]-E\left[Z_{n} \mid X_{0}=i\right]
$$

This expectation problem is combined with the former two problems: maximum statistics and minimum statistics. 
However, in general

$$
E\left[h\left(Y_{n}, Z_{n}\right) \mid X_{0}=i\right] \neq h\left(E\left[Y_{n} \mid X_{0}=i\right], E\left[Z_{n} \mid X_{0}=i\right]\right)
$$

We imbed this problem into the class of two-parametrized problems:

$$
u_{n}(i ; \lambda, \mu)=E\left[h\left(\lambda \vee Y_{n}, \mu \wedge Z_{n}\right) \mid X_{0}=i\right] \quad m \leq \lambda, \mu \leq M
$$

Then for a sufficiently small value $\tilde{\lambda}$ of $\lambda$ and a sufficiently large value $\tilde{\mu}$ of $\mu$ we have

$$
u_{n}(i ; \tilde{\lambda}, \tilde{\mu})=u_{n}(i) .
$$

In particular,

$$
u_{n}(i ; m, M)=u_{n}(i)
$$

We get the recurrence equation:

$$
\begin{aligned}
& u_{0}(i ; \lambda, \mu)=h(\lambda \vee f(i), \mu \wedge f(i)) \\
& u_{n}(i ; \lambda, \mu)=\sum_{j=1}^{N} u_{n-1}(j ; \lambda \vee f(i), \mu \wedge f(i)) p(j \mid i) \quad n \geq 1
\end{aligned}
$$

On the other hand, we imbed the problem (12) into the class of backward problems:

$$
u^{n-k+1}(i ; \lambda, \mu)=E\left[h\left(\lambda \vee Y_{k, n}, \mu \wedge Z_{k, n}\right) \mid X_{k}=i\right] \quad 0 \leq k \leq n, \quad m \leq \lambda, \mu \leq M .
$$

We have

$$
u^{n+1}(i ; m, M)=u_{n}(i)
$$

and the backward recurrence equation:

$$
\begin{aligned}
u^{1}(i ; \lambda, \mu) & =h(\lambda \vee f(i), \mu \wedge f(i)) \\
u^{n-k+1}(i ; \lambda, \mu) & =\sum_{j=1}^{N} u^{n-k}(j ; \lambda \vee f(i), \mu \wedge f(i)) p(j \mid i) \quad 0 \leq k \leq n-1
\end{aligned}
$$

\subsection{Ratio Statistics}

Further, we define

$$
S_{n}=f\left(X_{0}\right)+f\left(X_{1}\right)+\cdots+f\left(X_{n}\right) \quad n \geq 0
$$

and

$$
T_{n}=g\left(X_{0}\right)+g\left(X_{1}\right)+\cdots+g\left(X_{n}\right) \quad n \geq 0 .
$$

Second, we consider for any fixed $n \geq 0$ the conditional expected value :

$$
v_{n}(i)=E\left[\frac{S_{n}}{T_{n}} \mid X_{0}=i\right]
$$


(See Sniedovich $(1989,1992)$ for optimization problem.) We note that

$$
E\left[\frac{S_{n}}{T_{n}} \mid X_{0}=i\right] \neq \frac{E\left[S_{n} \mid X_{0}=i\right]}{E\left[T_{n} \mid X_{0}=i\right]} .
$$

Now, we imbed this problem into the class of two-parametrized problems:

$$
\begin{aligned}
& v_{k}(i ; \lambda, \mu)=E\left[\frac{\lambda+S_{k}}{\mu+T_{k}} \mid X_{0}=i\right] \quad 0 \leq k \leq n \\
& \lambda \in\left[\underline{\lambda}_{k}, \bar{\lambda}_{k}\right], \quad \mu \in\left[\underline{\mu}_{k}, \bar{\mu}_{k}\right]
\end{aligned}
$$

where

$$
\begin{gathered}
\underline{\lambda}_{k}=(n-k) \underline{f} \quad \bar{\lambda}_{k}=(n-k) \bar{f} \quad 0 \leq k \leq n \\
\underline{\mu}_{k}=(n-k) \underline{g} \quad \bar{\mu}_{k}=(n-k) \bar{g} \quad 0 \leq k \leq n \\
\underline{f}=\min _{s \in S} f(s), \quad \bar{f}=\max _{s \in S} f(s) \\
\underline{g}=\min _{s \in S} g(s), \quad \bar{g}=\max _{s \in S} g(s) .
\end{gathered}
$$

Then we have

$$
v_{n}(i ; 0,0)=v_{n}(i)
$$

and the forward recurrence equation:

$$
\begin{gathered}
v_{0}(i ; \lambda, \mu)=\frac{\lambda+f(i)}{\mu+g(i)} \quad \lambda \in\left[\underline{\lambda}_{0}, \bar{\lambda}_{0}\right], \quad \mu \in\left[\underline{\mu}_{0}, \bar{\mu}_{0}\right] \\
v_{k}(i ; \lambda, \mu)=\sum_{j=1}^{N} v_{k-1}(j ; \lambda+f(i), \mu+g(i)) p(j \mid i) \quad 1 \leq k \leq n \\
\lambda \in\left[\underline{\lambda}_{k}, \bar{\lambda}_{k}\right], \quad \mu \in\left[\underline{\mu}_{k}, \bar{\mu}_{k}\right] .
\end{gathered}
$$

On the other hand, we imbed the problem (13) into the class of backward problems:

$$
\begin{aligned}
v^{n-k+1}(i ; \lambda, \mu)=E\left[\frac{\lambda+S_{k, n}}{\mu+T_{k, n}} \mid X_{k}=i\right] & 0 \leq k \leq n \\
\lambda \in[k \underline{f}, k \bar{f}], & \mu \in[k \underline{f}, k \bar{f}]
\end{aligned}
$$

where

$$
S_{k, n}=f\left(X_{k}\right)+f\left(X_{k+1}\right)+\cdots+f\left(X_{n}\right) \quad 0 \leq k \leq n
$$

and

$$
T_{k, n}=g\left(X_{k}\right)+g\left(X_{k+1}\right)+\cdots+g\left(X_{n}\right) \quad 0 \leq k \leq n .
$$

We have

$$
v^{n+1}(i ; 0,0)=v_{n}(i)
$$


and the backward recurrence equation:

$$
\begin{gathered}
v^{1}(i ; \lambda, \mu)=\frac{\lambda+f(i)}{\mu+g(i)} \quad \lambda \in[n \underline{f}, n \bar{f}], \quad \mu \in[n \underline{g}, n \bar{g}] \\
v^{n-k+1}(i ; \lambda, \mu)=\sum_{j=1}^{N} v^{n-k}(j ; \lambda+f(i), \mu+g(i)) p(j \mid i) \quad 0 \leq k \leq n-1 \\
\lambda \in[k \underline{f}, k \bar{f}], \quad \mu \in[k \underline{g}, k \bar{g}] .
\end{gathered}
$$

\subsection{Variance Statistics}

Finally, we consider for any fixed $n \geq 1$ the conditional expected value :

$$
E\left\{\frac{1}{n}\left[\sum_{k=0}^{n-1}\left(f_{k}-\mu\right)^{2}\right] \mid X_{0}=i\right\}
$$

where

$$
\mu=\frac{1}{n} \sum_{k=0}^{n-1} f_{k}, \quad f_{k}=f\left(X_{k}\right)
$$

(See Sniedovich(1983, 1992) for deterministic optimization problem.) Note that

$$
\frac{1}{n}\left[\sum_{k=0}^{n-1}\left(f_{k}-\mu\right)^{2}\right]=\frac{1}{n} \sum_{k=0}^{n-1} f_{k}^{2}-\left(\frac{1}{n} \sum_{k=0}^{n-1} f_{k}\right)^{2} .
$$

Then we calculate the expected value multiplied by $n^{2}$ instead of (14):

$$
n^{2} E\left\{\frac{1}{n}\left[\sum_{k=0}^{n-1}\left(f_{k}-\mu\right)^{2}\right] \mid X_{0}=i\right\} .
$$

That is, we set

$$
v_{n}(i)=E\left[n \sum_{k=0}^{n-1} f_{k}^{2}-\left(\sum_{k=0}^{n-1} f_{k}\right)^{2} \mid X_{0}=i\right] .
$$

Now, we imbed this problem into the class of one-parametrized problems:

$$
\begin{gathered}
v_{k}(i ; \lambda)=E\left[n \sum_{l=0}^{k-1} f_{l}^{2}-\left(\lambda+\sum_{l=0}^{k-1} f_{l}\right)^{2} \mid X_{0}=i\right] \\
1 \leq k \leq n \quad \lambda \in\left[\underline{\lambda}_{k}, \bar{\lambda}_{k}\right] .
\end{gathered}
$$

We have

$$
v_{n}(i ; 0)=v_{n}(i)
$$

and the forward recurrence equation:

$$
\begin{aligned}
v_{1}(i ; \lambda)= & n f^{2}(i)-(\lambda+f(i))^{2} \quad \lambda \in\left[\underline{\lambda}_{1}, \bar{\lambda}_{1}\right] \\
v_{k}(i ; \lambda)= & n f^{2}(i)+\sum_{j=1}^{N} v_{k-1}(j ; \lambda+f(i)) p(j \mid i) \\
& 2 \leq k \leq n \quad \lambda \in\left[\underline{\lambda}_{k}, \bar{\lambda}_{k}\right] .
\end{aligned}
$$


Then the desired expected value of (14) is given by

$$
\frac{1}{n^{2}} v_{n}(i ; 0)=\frac{1}{n^{2}} v_{n}(i) \text {. }
$$

On the other hand, we imbed the problem (15) into the class of backward problems:

$$
\begin{aligned}
v^{n-k}(i ; \lambda)=E\left[n \sum_{l=k}^{n-1} f_{l}^{2}-\left(\lambda+\sum_{l=k}^{n-1} f_{l}\right)^{2} \mid X_{k}=i\right] \\
0 \leq k \leq n-1 \quad \lambda \in[k \underline{f}, k \bar{f}] .
\end{aligned}
$$

We have

$$
v^{n}(i ; 0)=v_{n}(i)
$$

and the backward recurrence equation:

$$
\begin{aligned}
v^{1}(i ; \lambda) & =n f^{2}(i)-(\lambda+f(i))^{2} \quad \lambda \in[(n-1) \underline{f},(n-1) \bar{f}] \\
v^{n-k}(i ; \lambda) & =n f^{2}(i)+\sum_{j=1}^{N} v^{n-k-1}(j ; \lambda+f(i)) p(j \mid i) \\
& 0 \leq k \leq n-2 \quad \lambda \in[k \underline{f}, k \bar{f}] .
\end{aligned}
$$

\section{Associative/Nonassociative Binary Relations}

We illustrates both classes of typical associative binary relations and of nonassocitive ones in Markov decision process and/or stochastic dynamic programming. Since the correspondence between each binary relation below and the resulting objective functions of optimization problems discussed in the references is straightforward, we omit specifying it.

First we have the following examples of associative binary relations $\circ$ with leftidentity in parenthesis.

$$
\begin{aligned}
\text { Example } 1 \quad \circ: R^{1} \times R^{1} \rightarrow R^{1} & \\
<\text { additive }> & x \circ y=x+y \quad(\tilde{x}=0) \\
<\text { multiplicative }> & x \circ y=x y \quad(\tilde{x}=1) \\
<\text { terminal }> & x \circ y=y \quad(\tilde{x}=\text { any real number }) . \\
<g-\text { additive }> & x \circ y=g^{-1}(g(x)+g(y)) \quad\left(\tilde{x}=g^{-1}(0)\right) \\
<g-\text { multiplicative }> & x \circ y=g^{-1}(g(x) g(y)) \quad\left(\tilde{x}=g^{-1}(1)\right)
\end{aligned}
$$

where $g: R^{1} \rightarrow R^{1}$ is onto continuous and strictly increasing.

$$
\begin{aligned}
& \text { Example } 2 \quad \circ:[0,1] \times[0,1] \rightarrow[0,1] \\
& \qquad \begin{aligned}
<\text { maximum }>\quad x \circ y=x \vee y \quad(\tilde{x}=0) \\
<\text { minimum }>\quad x \circ y=x \wedge y \quad(\tilde{x}=1)
\end{aligned}
\end{aligned}
$$


Example $3 \quad \circ: R^{1} \times R^{1}-\{(x, y) \mid x y \neq-1\} \rightarrow R^{1}$

$$
x \circ y=\frac{x+y}{1+x y} \quad(\tilde{x}=1) \text {. }
$$

Example $4 \quad \circ: R^{1} \times R^{1}-\{(x, y) \mid x+y \neq 0\} \rightarrow R^{1}$

$$
x \circ y=\frac{1+x y}{x+y} \quad(\tilde{x}=1) .
$$

Example $5 \quad \circ: R_{+}^{1} \times R_{+}^{1} \rightarrow R_{+}^{1}$

$$
<p-\text { normed }>\quad x \circ y=\left(x^{p}+y^{p}\right)^{1 / p} \quad(\tilde{x}=0)
$$

where

$$
p>0 \text {. }
$$

On the other hand, the following are examples of nonassociative binary relation 0 .

Example $6 \quad \circ: R^{1} \times R^{1} \rightarrow R^{1}$

$$
\begin{aligned}
<\text { multiplicatively additive }> & x \circ y=x+x y \\
<\text { discounted }> & x \circ y=x+\beta y
\end{aligned}
$$

where

$$
\beta \neq 1 \text {. }
$$

Example $7 \quad \circ:(0, \infty) \times(0, \infty) \rightarrow(0, \infty)$

$$
\begin{array}{ll}
<\text { backward exponential }> & x \circ y=x^{y} \\
<\text { forward exponential }> & x \circ y=y^{x}
\end{array}
$$

\section{Examples}

In this section we specify three-stage statistics and calculate the expected values of them on two-state Markov chain.

\subsection{Three-stage statistics}

In this subsection we specify explicit forms for three-stage statistics. The $n$-stage statistics are straightforward.

Example 1

$$
\begin{aligned}
<\text { additive }> & E\left[R_{0}+R_{1}+R_{2}+R_{3} \mid X_{0}=i\right] \\
<\text { multiplicative }> & E\left[R_{0} R_{1} R_{2} R_{3} \mid X_{0}=i\right] \\
<\text { terminal }> & E\left[R_{3} \mid X_{0}=i\right] \\
<g-\text { additive }> & E\left[g^{-1}\left(g\left(R_{0}\right)+g\left(R_{1}\right)+g\left(R_{2}\right)+g\left(R_{3}\right)\right) \mid X_{0}=i\right] \\
<g-\text { multiplicative }> & E\left[g^{-1}\left(g\left(R_{0}\right) g\left(R_{1}\right) g\left(R_{2}\right) g\left(R_{3}\right)\right) \mid X_{0}=i\right]
\end{aligned}
$$


where $g: R^{1} \rightarrow R^{1}$ is onto continuous and strictly increasing.

Example 2

$$
\begin{array}{ll}
<\text { maximum }> & E\left[R_{0} \vee R_{1} \vee R_{2} \vee R_{3} \mid X_{0}=i\right] \\
<\text { minimum }> & E\left[R_{0} \wedge R_{1} \wedge R_{2} \wedge R_{3} \mid X_{0}=i\right] .
\end{array}
$$

Example 3

$$
E\left[\frac{R_{0}+R_{1}+R_{2}+R_{3}+R_{0} R_{1} R_{2}+R_{0} R_{1} R_{3}+R_{0} R_{2} R_{3}+R_{1} R_{2} R_{3}}{1+R_{0} R_{1}+R_{0} R_{2}+R_{0} R_{3}+R_{1} R_{2}+R_{1} R_{3}+R_{2} R_{3}+R_{0} R_{1} R_{2} R_{3}} \mid X_{0}=i\right]
$$

Example 4

$$
E\left[\frac{1+R_{0} R_{1}+R_{0} R_{2}+R_{0} R_{3}+R_{1} R_{2}+R_{1} R_{3}+R_{2} R_{3}+R_{0} R_{1} R_{2} R_{3}}{R_{0}+R_{1}+R_{2}+R_{3}+R_{0} R_{1} R_{2}+R_{0} R_{1} R_{3}+R_{0} R_{2} R_{3}+R_{1} R_{2} R_{3}} \mid X_{0}=i\right] .
$$

\section{Example 5}

$$
<p \text {-normed }>\quad E\left[\left(R_{0}^{p}+R_{1}^{p}+R_{2}^{p}+R_{3}^{p}\right)^{1 / p} \mid X_{0}=i\right]
$$

where

$$
p>0 .
$$

On the other hand, the following are examples of nonassociative statistics.

Example 6

$$
\begin{gathered}
<\text { multiplicatively additive }>E\left[R_{0}+R_{0} R_{1}+R_{0} R_{1} R_{2}+R_{0} R_{1} R_{2} R_{3} \mid X_{0}=i\right] \\
<\text { discounted }>E\left[R_{0}+\beta R_{1}+\beta^{2} R_{2}+\beta^{3} R_{3} \mid X_{0}=i\right]
\end{gathered}
$$

where

$$
\beta \neq 1 \text {. }
$$

Example 7 (See also Golmb $(1975,1980)$ for the corresponding deterministic optimization problems.)

$$
\begin{aligned}
& <\text { backward exponential > } \\
& E\left[\left(\left(R_{0}^{R_{1}}\right)^{R_{2}}\right)^{R_{3}} \mid X_{0}=i\right] \\
& <\text { forward exponential }> \\
& E\left[R_{3}^{R_{2}^{R_{1}^{R_{0}}}} \mid X_{0}=i\right] .
\end{aligned}
$$

As compound statistics, we consider the expected value of variance and ratio statistics as follows : 
Example 8

$$
\begin{array}{ll}
<\text { variance }> & E\left[\frac{1}{4} \sum_{i=0}^{3}\left(R_{i}-\bar{R}\right)^{2} \mid X_{0}=i\right] \quad \bar{R}=\frac{1}{4} \sum_{i=0}^{3} R_{i} \\
<\text { ratio }> & E\left[\frac{R_{0}+R_{1}+R_{2}+R_{3}}{R_{0}^{2}+R_{1}^{2}+R_{2}^{2}+R_{3}^{2}} \mid X_{0}=i\right] .
\end{array}
$$

\subsection{Two-state Markov Chain}

Now we consider a simple Markov chain on state space $S=\left\{s_{1}, s_{2}\right\}$ with the following numerical data :

\begin{tabular}{c|cc}
$r\left(s_{1}\right)=2$, & \multicolumn{2}{c}{$r\left(s_{2}\right)=-1$} \\
\hline$x_{t} \backslash x_{t+1}$ & $s_{1}$ & $s_{2}$ \\
\hline$s_{1}$ & $1 / 4$ & $3 / 4$ \\
$s_{2}$ & $1 / 2$ & $1 / 2$ \\
\hline
\end{tabular}

\subsection{Simple Statistics}

First we consider the expected value of multilpicative, minimum, maximum and terminal statistics.

\begin{tabular}{|c|c|c|c|c|c|}
\hline history(state, trans. prob) & path & mult. & $\min$. & $\max$ & ter. \\
\hline $\begin{array}{lllllll}s_{1} & 1 / 4 & s_{1} & 1 / 4 & s_{1} & 1 / 4 & s_{1}\end{array}$ & $1 / 64$ & 16 & 2 & 2 & 2 \\
\hline $\begin{array}{lllllll}s_{1} & 1 / 4 & s_{1} & 1 / 4 & s_{1} & 3 / 4 & s_{2}\end{array}$ & $3 / 64$ & -8 & -1 & 2 & -1 \\
\hline $\begin{array}{lllllll}s_{1} & 1 / 4 & s_{1} & 3 / 4 & s_{2} & 1 / 2 & s_{1}\end{array}$ & $6 / 64$ & -8 & -1 & 2 & 2 \\
\hline $\begin{array}{lllllll}s_{1} & 1 / 4 & s_{1} & 3 / 4 & s_{2} & 1 / 2 & s_{2}\end{array}$ & $6 / 64$ & 4 & -1 & 2 & -1 \\
\hline $\begin{array}{lllllll}s_{1} & 3 / 4 & s_{2} & 1 / 2 & s_{1} & 1 / 4 & s_{1}\end{array}$ & $6 / 64$ & -8 & -1 & 2 & 2 \\
\hline $\begin{array}{lllllll}s_{1} & 3 / 4 & s_{2} & 1 / 2 & s_{1} & 3 / 4 & s_{2}\end{array}$ & $18 / 64$ & 4 & -1 & 2 & -1 \\
\hline $\begin{array}{lllllll}s_{1} & 3 / 4 & s_{2} & 1 / 2 & s_{2} & 1 / 2 & s_{1}\end{array}$ & $12 / 64$ & 4 & -1 & 2 & 2 \\
\hline $\begin{array}{lllllll}s_{1} & 3 / 4 & s_{2} & 1 / 2 & s_{2} & 1 / 2 & s_{2} \\
\end{array}$ & $12 / 64$ & 4 & -1 & 2 & -1 \\
\hline expected value from $s_{1}$ & & $1 / 4$ & $-61 / 64$ & 2 & $11 / 64$ \\
\hline $\begin{array}{lllllll}s_{2} & 1 / 2 & s_{1} & 1 / 4 & s_{1} & 1 / 4 & s_{1}\end{array}$ & $2 / 64$ & -8 & -1 & 2 & 2 \\
\hline $\begin{array}{lllllll}s_{2} & 1 / 2 & s_{1} & 1 / 4 & s_{1} & 3 / 4 & s_{2}\end{array}$ & $6 / 64$ & 4 & -1 & 2 & -1 \\
\hline $\begin{array}{lllllll}s_{2} & 1 / 2 & s_{1} & 3 / 4 & s_{2} & 1 / 2 & s_{1}\end{array}$ & $12 / 64$ & 4 & -1 & 2 & 2 \\
\hline $\begin{array}{lllllll}s_{2} & 1 / 2 & s_{1} & 3 / 4 & s_{2} & 1 / 2 & s_{2}\end{array}$ & $12 / 64$ & -2 & -1 & 2 & -1 \\
\hline $\begin{array}{lllllll}s_{2} & 1 / 2 & s_{2} & 1 / 2 & s_{1} & 1 / 4 & s_{1}\end{array}$ & $4 / 64$ & 4 & -1 & 2 & 2 \\
\hline $\begin{array}{lllllll}s_{2} & 1 / 2 & s_{2} & 1 / 2 & s_{1} & 3 / 4 & s_{2}\end{array}$ & $12 / 64$ & 4 & -1 & 2 & -1 \\
\hline $\begin{array}{lllllll}s_{2} & 1 / 2 & s_{2} & 1 / 2 & s_{2} & 1 / 2 & s_{1}\end{array}$ & $8 / 64$ & -2 & -1 & 2 & 2 \\
\hline $\begin{array}{lllllll}s_{2} & 1 / 2 & s_{2} & 1 / 2 & s_{2} & 1 / 2 & s_{2} \\
\end{array}$ & $8 / 64$ & 1 & -1 & -1 & -1 \\
\hline expected value from $s_{2}$ & & $1 / 4$ & -1 & $13 / 8$ & $7 / 32$ \\
\hline
\end{tabular}

Then the direct computation yields Table 1 as follows :

(where multi. = multiplication, $\min .=\operatorname{minimum}, \max .=\operatorname{maximum}$, ter. $=$ terminal)

Table 1 : expected values of simple statistics from $s_{1}, s_{2}$ 
On the other hand, the forward recurrence equation for multiplicative problem

$$
\begin{aligned}
& u_{0}(i ; \lambda)=\lambda r(i) \\
& u_{n}(i ; \lambda)=\sum_{j=1}^{2} u_{n-1}(j ; \lambda r(i)) p(j \mid i) \quad n=1,2,3
\end{aligned}
$$

yields the solution as follows :

$$
\begin{array}{llll}
u_{0}\left(s_{1} ; \lambda\right)=2 \lambda & u_{1}\left(s_{1} ; \lambda\right)=-\frac{1}{2} \lambda & u_{2}\left(s_{1} ; \lambda\right)=-\lambda & u_{3}\left(s_{1} ; \lambda\right)=\frac{1}{4} \lambda \\
u_{0}\left(s_{2} ; \lambda\right)=-\lambda & u_{1}\left(s_{2} ; \lambda\right)=-\frac{1}{2} \lambda & u_{2}\left(s_{2} ; \lambda\right)=\frac{1}{2} \lambda & u_{3}\left(s_{2} ; \lambda\right)=\frac{1}{4} \lambda
\end{array}
$$

Therefore we get the desired expected values

$$
u_{3}\left(s_{1}\right)=u_{3}\left(s_{1} ; 1\right)=\frac{1}{4}, \quad u_{3}\left(s_{2}\right)=u_{3}\left(s_{2} ; 1\right)=\frac{1}{4} .
$$

For the minimun problem, the forward recurrence equation

$$
\begin{aligned}
& u_{0}(i ; \lambda)=\lambda \wedge r(i) \\
& u_{n}(i ; \lambda)=\sum_{j=1}^{2} u_{n-1}(j ; \lambda \wedge(i)) p(j \mid i) \quad n=1,2,3
\end{aligned}
$$

yields

$$
\begin{aligned}
u_{0}\left(s_{1} ; \lambda\right)=\lambda \wedge 2 \\
u_{0}\left(s_{2} ; \lambda\right)=\lambda \wedge(-1) \\
u_{1}\left(s_{1} ; \lambda\right)=\frac{1}{4}(\lambda \wedge 2)+\frac{3}{4}(\lambda \wedge(-1)) \\
u_{1}\left(s_{2} ; \lambda\right)=\lambda \wedge(-1) \\
u_{2}\left(s_{1} ; \lambda\right)=\frac{1}{16}(\lambda \wedge 2)+\frac{15}{16}(\lambda \wedge(-1)) \\
u_{2}\left(s_{2} ; \lambda\right)=\lambda \wedge(-1) \\
u_{3}\left(s_{1} ; \lambda\right)=\frac{1}{64}(\lambda \wedge 2)+\frac{63}{64}(\lambda \wedge(-1)) \\
u_{3}\left(s_{2} ; \lambda\right)=\lambda \wedge(-1) .
\end{aligned}
$$

Thus we have the desired expected values

$$
u_{3}\left(s_{1}\right)=u_{3}\left(s_{1} ; 2\right)=-\frac{61}{64}, \quad u_{3}\left(s_{2}\right)=u_{3}\left(s_{2} ; 2\right)=-1
$$


For the maximum problem, the forward recurrence equation

$$
\begin{aligned}
& u_{0}(i ; \lambda)=\lambda \vee r(i) \\
& u_{n}(i ; \lambda)=\sum_{j=1}^{2} u_{n-1}(j ; \lambda \vee(i)) p(j \mid i) \quad n=1,2,3
\end{aligned}
$$

yields

$$
\begin{aligned}
u_{0}\left(s_{1} ; \lambda\right) & =\lambda \vee 2 \\
u_{0}\left(s_{2} ; \lambda\right) & =\lambda \vee(-1) \\
u_{1}\left(s_{1} ; \lambda\right) & =\lambda \vee 2 \\
u_{1}\left(s_{2} ; \lambda\right) & =\frac{1}{2}(\lambda \vee 2)+\frac{1}{2}(\lambda \vee(-1)) \\
u_{2}\left(s_{1} ; \lambda\right) & =\lambda \vee 2 \\
u_{2}\left(s_{2} ; \lambda\right) & =\frac{3}{4}(\lambda \vee 2)+\frac{1}{4}(\lambda \vee(-1)) \\
u_{3}\left(s_{1} ; \lambda\right) & =\lambda \vee 2 \\
u_{3}\left(s_{2} ; \lambda\right) & =\frac{7}{8}(\lambda \vee 2)+\frac{1}{8}(\lambda \vee(-1))
\end{aligned}
$$

Thus we have the desired expected values

$$
u_{3}\left(s_{1}\right)=u_{3}\left(s_{1} ;-1\right)=2, \quad u_{3}\left(s_{2}\right)=u_{3}\left(s_{2} ;-1\right)=\frac{13}{8} .
$$

For the terminal problem, the forward recurrence equation

$$
\begin{aligned}
& u_{0}(i ; \lambda)=r(i) \\
& u_{n}(i ; \lambda)=\sum_{j=1}^{2} u_{n-1}(j ; r(i)) p(j \mid i) \quad n=1,2,3
\end{aligned}
$$

yields

$$
\begin{array}{lcll}
u_{0}\left(s_{1} ; \lambda\right)=2 & u_{1}\left(s_{1} ; \lambda\right)=-\frac{1}{4} & u_{2}\left(s_{1} ; \lambda\right)=\frac{5}{16} & u_{3}\left(s_{1} ; \lambda\right)=\frac{11}{64} \\
u_{0}\left(s_{2} ; \lambda\right)=-1 & u_{1}\left(s_{2} ; \lambda\right)=\frac{1}{2} & u_{2}\left(s_{2} ; \lambda\right)=\frac{1}{8} & u_{3}\left(s_{2} ; \lambda\right)=\frac{7}{32}
\end{array}
$$

Thus we have the desired expected values

$$
u_{3}\left(s_{1}\right)=u_{3}\left(s_{1} ; \lambda\right)=\frac{11}{64}, \quad u_{3}\left(s_{2}\right)=u_{3}\left(s_{2} ; \lambda\right)=\frac{7}{32} .
$$




\subsection{Variance}

Second, we illustrate how to iteratively calculate the expected value of variance statistics. We remak that

$$
E\left[\frac{1}{4} \sum_{i=0}^{3}\left(R_{i}-\bar{R}\right)^{2} \mid X_{0}=i\right]=E\left[\frac{1}{4} \sum_{i=0}^{3} R_{i}^{2} \mid X_{0}=i\right]-E\left[\left(\frac{1}{4} \sum_{i=0}^{3} R_{i}\right)^{2} \mid X_{0}=i\right]
$$

where

$$
\bar{R}=\frac{1}{4} \sum_{i=0}^{3} R_{i}
$$

Thus we apply the famous formula:

$$
\text { variance }=\text { mean square }- \text { square mean } .
$$

\begin{tabular}{|c|c|c|c|c|c|}
\hline history(state, trans. prob) & path & sum & sq. mean & mean sq. & variance \\
\hline $\begin{array}{lllllll}s_{1} & 1 / 4 & s_{1} & 1 / 4 & s_{1} & 1 / 4 & s_{1}\end{array}$ & $1 / 64$ & 8 & 4 & 4 & 0 \\
\hline $\begin{array}{lllllll}s_{1} & 1 / 4 & s_{1} & 1 / 4 & s_{1} & 3 / 4 & s_{2}\end{array}$ & $3 / 64$ & 5 & $25 / 16$ & $13 / 4$ & $27 / 16$ \\
\hline $\begin{array}{lllllll}s_{1} & 1 / 4 & s_{1} & 3 / 4 & s_{2} & 1 / 2 & s_{1}\end{array}$ & $6 / 64$ & 5 & $25 / 16$ & $13 / 4$ & $27 / 16$ \\
\hline $\begin{array}{lllllll}s_{1} & 1 / 4 & s_{1} & 3 / 4 & s_{2} & 1 / 2 & s_{2}\end{array}$ & $6 / 64$ & 2 & $1 / 4$ & $10 / 4$ & $9 / 4$ \\
\hline $\begin{array}{lllllll}s_{1} & 3 / 4 & s_{2} & 1 / 2 & s_{1} & 1 / 4 & s_{1}\end{array}$ & $6 / 64$ & 5 & $25 / 16$ & $13 / 4$ & $27 / 16$ \\
\hline $\begin{array}{lllllll}s_{1} & 3 / 4 & s_{2} & 1 / 2 & s_{1} & 3 / 4 & s_{2}\end{array}$ & $18 / 64$ & 2 & $1 / 4$ & $10 / 4$ & $9 / 4$ \\
\hline $\begin{array}{lllllll}s_{1} & 3 / 4 & s_{2} & 1 / 2 & s_{2} & 1 / 2 & s_{1}\end{array}$ & $12 / 64$ & 2 & $1 / 4$ & $10 / 4$ & $9 / 4$ \\
\hline $\begin{array}{lllllll}s_{1} & 3 / 4 & s_{2} & 1 / 2 & s_{2} & 1 / 2 & s_{2}\end{array}$ & $12 / 64$ & -1 & $1 / 16$ & $7 / 4$ & $27 / 16$ \\
\hline expected value from $s_{1}$ & & $143 / 64$ & $595 / 1024$ & $655 / 256$ & $2025 / 1024$ \\
\hline $\begin{array}{llllllll}s_{2} & 1 / 2 & s_{1} & 1 / 4 & s_{1} & 1 / 4 & s_{1}\end{array}$ & $2 / 64$ & 5 & $25 / 16$ & $13 / 4$ & $27 / 16$ \\
\hline $\begin{array}{lllllll}s_{2} & 1 / 2 & s_{1} & 1 / 4 & s_{1} & 3 / 4 & s_{2}\end{array}$ & $6 / 64$ & 2 & $1 / 4$ & $10 / 4$ & $9 / 4$ \\
\hline $\begin{array}{lllllll}s_{2} & 1 / 2 & s_{1} & 3 / 4 & s_{2} & 1 / 2 & s_{1}\end{array}$ & $12 / 64$ & 2 & $1 / 4$ & $10 / 4$ & $9 / 4$ \\
\hline $\begin{array}{lllllll}s_{2} & 1 / 2 & s_{1} & 3 / 4 & s_{2} & 1 / 2 & s_{2}\end{array}$ & $12 / 64$ & -1 & $1 / 16$ & $7 / 4$ & $27 / 16$ \\
\hline $\begin{array}{lllllll}s_{2} & 1 / 2 & s_{2} & 1 / 2 & s_{1} & 1 / 4 & s_{1}\end{array}$ & $4 / 64$ & 2 & $1 / 4$ & $10 / 4$ & $9 / 4$ \\
\hline$s_{2} \quad 1 / 2 \quad s_{2} \quad 1 / 2 \quad s_{1} \quad 3 / 4 \quad s_{2}$ & $12 / 64$ & -1 & $1 / 16$ & $7 / 4$ & $27 / 16$ \\
\hline 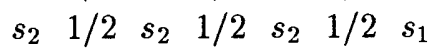 & $8 / 64$ & -1 & $1 / 16$ & $7 / 4$ & $27 / 16$ \\
\hline $\begin{array}{lllllll}s_{2} & 1 / 2 & s_{2} & 1 / 2 & s_{2} & 1 / 2 & s_{2}\end{array}$ & $8 / 64$ & -4 & 1 & 1 & 0 \\
\hline expected value from $s_{2}$ & & $-5 / 32$ & $149 / 512$ & $251 / 128$ & $855 / 512$ \\
\hline
\end{tabular}

Then the direct computation yields Table 2 as follows :

Table 2: expected value of variance from $s_{1}, s_{2}$

First, we consider the problem multiplied by $4^{2}$ as follows :

$$
\begin{aligned}
& 4^{2} E\left[\frac{1}{4} \sum_{i=0}^{3}\left(R_{i}-\bar{R}\right)^{2} \mid X_{0}=i\right] \\
= & E\left[4 R_{0}^{2}+4 R_{1}^{2}+4 R_{2}^{2}+4 R_{3}^{2}-\left(R_{0}+R_{1}+R_{2}+R_{3}\right)^{2} \mid X_{0}=i\right]
\end{aligned}
$$


Imbedding this problem into the family of parametrized ones with $\lambda$ :

$$
\begin{aligned}
& u_{n}(i ; \lambda) \\
= & E\left[4 R_{0}^{2}+4 R_{1}^{2}+\cdots+4 R_{n-1}^{2}-\left(\lambda+R_{0}+R_{1}+\cdots+R_{n-1}\right)^{2} \mid X_{0}=i\right] \\
i & =1,2 \quad n=0,1,2,3,4
\end{aligned}
$$

we have the forward recurrence equation :

$$
\begin{aligned}
& u_{0}(i ; \lambda)=-\lambda^{2} \\
& u_{n}(i ; \lambda)=4 r(i)^{2}+\sum_{j=1}^{2} u_{n-1}(j ; \lambda+r(i)) p(j \mid i) \quad n=1,2,3 .
\end{aligned}
$$

Second, solving this equation, we obtain the following expressions :

$$
\begin{gathered}
u_{0}\left(s_{1} ; \lambda\right)=-\lambda^{2} \\
u_{0}\left(s_{2} ; \lambda\right)=-\lambda^{2} \\
u_{1}\left(s_{1} ; \lambda\right)=4 \cdot 2^{2}-(\lambda+2)^{2} \\
u_{1}\left(s_{2} ; \lambda\right)=4(-1)^{2}-(\lambda-1)^{2} \\
u_{2}\left(s_{1} ; \lambda\right)=5 \cdot 2^{2}+3(-1)^{2}-\frac{1}{4}(\lambda+4)^{2}-\frac{3}{4}(\lambda+1)^{2} \\
u_{2}\left(s_{2} ; \lambda\right)=2 \cdot 2^{2}+6(-1)^{2}-\frac{1}{2}(\lambda+1)^{2}-\frac{1}{2}(\lambda-2)^{2} \\
u_{3}\left(s_{1} ; \lambda\right)=\frac{27}{4} \cdot 2^{2}+\frac{21}{4}(-1)^{2}-\frac{1}{4^{2}}(\lambda+6)^{2}-\frac{9}{4^{2}}(\lambda+3)^{2}-\frac{3}{2 \cdot 4} \lambda^{2} \\
u_{3}\left(s_{2} ; \lambda\right)=\frac{7}{2} \cdot 2^{2}+\frac{17}{2}(-1)^{2}-\frac{1}{2 \cdot 4}(\lambda+3)^{2}-\frac{5}{2 \cdot 4} \lambda^{2}-\frac{1}{2 \cdot 2}(\lambda-3)^{2} \\
u_{4}\left(s_{1} ; \lambda\right)=\frac{133}{4^{2}} \cdot 2^{2}+\frac{3 \cdot 41}{4^{2}}(-1)^{2}-\frac{1}{4^{3}}(\lambda+8)^{2}-\frac{15}{4^{3}}(\lambda+5)^{2} \\
-\frac{3^{2}}{4^{2}}(\lambda+2)^{2}-\frac{3}{4^{2}}(\lambda-1)^{2} \\
u_{4}\left(s_{2} ; \lambda\right)=\frac{11}{4 \cdot 2} \cdot 2^{2}+\frac{87}{8}(-1)^{2}-2 \cdot 4^{2}(\lambda+5)^{2}-\frac{11}{2 \cdot 4^{2}}(\lambda+2)^{2} \\
-\frac{1}{2}(\lambda-1)^{2}-\frac{1}{8}(\lambda-4)^{2}
\end{gathered}
$$

Finally, substituting $\lambda=0$, we have

$$
\begin{aligned}
u_{4}\left(s_{1} ; 0\right) & =\frac{133}{4^{2}} \cdot 2^{2}+\frac{3 \cdot 41}{4^{2}}(-1)^{2}-\frac{1}{4^{3}} \cdot 8^{2}-\frac{15}{4^{3}} \cdot 5^{2}-\frac{3^{2}}{4^{2}} \cdot 2^{2}-\frac{3}{4^{2}} \cdot(-1)^{2} \\
& =\frac{3^{4} \cdot 5^{2}}{2^{6}} \\
u_{4}\left(s_{2} ; 0\right) & =\frac{41}{4 \cdot 2} \cdot 2^{2}+\frac{87}{8}(-1)^{2}-\frac{1}{2 \cdot 4^{2}} \cdot 5^{2}-\frac{11}{2 \cdot 4^{2}} \cdot 2^{2}-\frac{1}{2} \cdot(-1)^{2}-\frac{1}{8} \cdot(-4)^{2} \\
& =\frac{5 \cdot 9 \cdot 19}{2^{5}} .
\end{aligned}
$$


Therefore we get the desired expected values

$$
\begin{aligned}
E\left[\frac{1}{4} \sum_{i=0}^{3}\left(R_{i}-\bar{R}\right)^{2} \mid X_{0}=s_{1}\right] & =\frac{1}{4^{2}} \cdot \frac{3^{4} \cdot 5^{2}}{2^{6}}=\frac{3^{4} \cdot 5^{2}}{2^{10}} \\
& =\frac{2025}{1024}=1.9775390625 \\
E\left[\frac{1}{4} \sum_{i=0}^{3}\left(R_{i}-\bar{R}\right)^{2} \mid X_{0}=s_{2}\right] & =\frac{1}{4^{2}} \cdot \frac{5 \cdot 9 \cdot 19}{2^{5}}=\frac{5 \cdot 9 \cdot 19}{2^{9}} \\
& =\frac{855}{512}=1.669921875 .
\end{aligned}
$$

\subsection{Ratio}

\begin{tabular}{|c|c|c|c|c|}
\hline history(state, trans. prob) & path & sum & sum sq. & ratio \\
\hline $\begin{array}{lllllll}s_{1} & 1 / 4 & s_{1} & 1 / 4 & s_{1} & 1 / 4 & s_{1}\end{array}$ & $1 / 64$ & 8 & 16 & $8 / 16$ \\
\hline 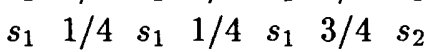 & $3 / 64$ & 5 & 13 & $5 / 13$ \\
\hline $\begin{array}{lllllll}s_{1} & 1 / 4 & s_{1} & 3 / 4 & s_{2} & 1 / 2 & s_{1}\end{array}$ & $6 / 64$ & 5 & 13 & $5 / 13$ \\
\hline $\begin{array}{lllllll}s_{1} & 1 / 4 & s_{1} & 3 / 4 & s_{2} & 1 / 2 & s_{2}\end{array}$ & $6 / 64$ & 2 & 10 & $2 / 10$ \\
\hline $\begin{array}{lllllll}s_{1} & 3 / 4 & s_{2} & 1 / 2 & s_{1} & 1 / 4 & s_{1}\end{array}$ & $6 / 64$ & 5 & 13 & $5 / 13$ \\
\hline $\begin{array}{lllllll}s_{1} & 3 / 4 & s_{2} & 1 / 2 & s_{1} & 3 / 4 & s_{2}\end{array}$ & $18 / 64$ & 2 & 10 & $2 / 10$ \\
\hline $\begin{array}{lllllll}s_{1} & 3 / 4 & s_{2} & 1 / 2 & s_{2} & 1 / 2 & s_{1}\end{array}$ & $12 / 64$ & 2 & 10 & $2 / 10$ \\
\hline $\begin{array}{lllllll}s_{1} & 3 / 4 & s_{2} & 1 / 2 & s_{2} & 1 / 2 & s_{2}\end{array}$ & $12 / 64$ & -1 & 7 & $-1 / 7$ \\
\hline expected value from $s_{1}$ & & $143 / 64$ & $655 / 256$ & $9969 / 58240$ \\
\hline $\begin{array}{lllllll}s_{2} & 1 / 2 & s_{1} & 1 / 4 & s_{1} & 1 / 4 & s_{1}\end{array}$ & $2 / 64$ & 5 & 13 & $5 / 13$ \\
\hline $\begin{array}{lllllll}s_{2} & 1 / 2 & s_{1} & 1 / 4 & s_{1} & 3 / 4 & s_{2}\end{array}$ & $6 / 64$ & 2 & 10 & $2 / 10$ \\
\hline $\begin{array}{lllllll}s_{2} & 1 / 2 & s_{1} & 3 / 4 & s_{2} & 1 / 2 & s_{1}\end{array}$ & $12 / 64$ & 2 & 10 & $2 / 10$ \\
\hline $\begin{array}{lllllll}s_{2} & 1 / 2 & s_{1} & 3 / 4 & s_{2} & 1 / 2 & s_{2}\end{array}$ & $12 / 64$ & -1 & 7 & $-1 / 7$ \\
\hline $\begin{array}{lllllll}s_{2} & 1 / 2 & s_{2} & 1 / 2 & s_{1} & 1 / 4 & s_{1}\end{array}$ & $4 / 64$ & 2 & 10 & $2 / 10$ \\
\hline $\begin{array}{lllllll}s_{2} & 1 / 2 & s_{2} & 1 / 2 & s_{1} & 3 / 4 & s_{2}\end{array}$ & $12 / 64$ & -1 & 7 & $-1 / 7$ \\
\hline $\begin{array}{lllllll}s_{2} & 1 / 2 & s_{2} & 1 / 2 & s_{2} & 1 / 2 & s_{1}\end{array}$ & $8 / 64$ & -1 & 7 & $-1 / 7$ \\
\hline $\begin{array}{lllllll}s_{2} & 1 / 2 & s_{2} & 1 / 2 & s_{2} & 1 / 2 & s_{2}\end{array}$ & $8 / 64$ & -4 & 4 & $-4 / 4$ \\
\hline expected value from $s_{2}$ & & $-5 / 32$ & $251 / 32$ & $-421 / 25480$ \\
\hline
\end{tabular}

Finally we consider the expected value of the following ratio statistics :

$$
E\left[\frac{R_{0}+R_{1}+R_{2}+R_{3}}{R_{0}^{2}+R_{1}^{2}+R_{2}^{2}+R_{3}^{2}} \mid X_{0}=i\right] .
$$

The direct computation yields Table 3 as follows :

Table 3 : expected value of a ratio from $s_{1}, s_{2}$

We consider the following ratio problem :

$$
u_{3}(i)=E\left[\frac{R_{0}+R_{1}+R_{2}+R_{3}}{R_{0}^{2}+R_{1}^{2}+R_{2}^{2}+R_{3}^{2}} \mid X_{0}=i\right]
$$


We imbed this problem into the family of two-parametrized ones with $\lambda, \mu$ :

$$
u_{n}(i ; \lambda, \mu)=E\left[\frac{\lambda+R_{0}+\cdots+R_{n}}{\mu+R_{0}^{2}+\cdots+R_{n}^{2}} \mid X_{0}=i\right] \quad i=1,2 \quad n=0,1,2,3
$$

Then we get the forward recurrence equation :

$$
\begin{aligned}
& u_{0}(i ; \lambda, \mu)=\frac{\lambda+r(i)}{\mu+r^{2}(i)} \\
& u_{n}(i ; \lambda, \mu)=\sum_{j=1}^{2} u_{n-1}\left(j ; \lambda+r(i), \mu+r^{2}(i)\right) p(j \mid i) \quad n=1,2,3 .
\end{aligned}
$$

Solving this equation, we obtain the following expressions :

$$
\begin{gathered}
u_{0}\left(s_{1} ; \lambda, \mu\right)=\frac{\lambda+2}{\mu+4} \\
u_{0}\left(s_{2} ; \lambda, \mu\right)=\frac{\lambda-1}{\mu+1} \\
u_{1}\left(s_{1} ; \lambda, \mu\right)=\frac{1}{4} \cdot \frac{\lambda+4}{\mu+8}+\frac{3}{4} \cdot \frac{\lambda+1}{\mu+5} \\
u_{1}\left(s_{2} ; \lambda, \mu\right)=\frac{1}{2} \cdot \frac{\lambda+1}{\mu+5}+\frac{1}{2} \cdot \frac{\lambda-2}{\mu+2} \\
u_{2}\left(s_{1} ; \lambda, \mu\right)=\frac{1}{4^{2}} \cdot \frac{\lambda+6}{\mu+12}+\frac{3^{2}}{4^{2}} \cdot \frac{\lambda+3}{\mu+9}+\frac{3}{2 \cdot 4} \cdot \frac{\lambda}{\mu+6} \\
u_{2}\left(s_{2} ; \lambda, \mu\right)=\frac{1}{4 \cdot 2} \cdot \frac{\lambda+3}{\mu+9}+\frac{5}{4 \cdot 2} \cdot \frac{\lambda}{\mu+6}+\frac{1}{2^{2}} \cdot \frac{\lambda-3}{\mu+3} \\
u_{3}\left(s_{1} ; \lambda, \mu\right)=\frac{1}{4^{3}} \cdot \frac{\lambda+8}{\mu+16}+\frac{3 \cdot 5}{4^{3}} \cdot \frac{\lambda+5}{\mu+13}+\frac{3 \cdot 6}{2 \cdot 4^{2}} \cdot \frac{\lambda+2}{\mu+10}+\frac{3}{2^{2} \cdot 4} \cdot \frac{\lambda-1}{\mu+7} \\
u_{3}\left(s_{2} ; \lambda, \mu\right)=\frac{1}{2 \cdot 4^{2}} \cdot \frac{\lambda+5}{\mu+13}+\frac{11}{2 \cdot 4^{2}} \cdot \frac{\lambda+2}{\mu+10}+\frac{1}{2} \cdot \frac{\lambda-1}{\mu+7}+\frac{1}{2^{3}} \cdot \frac{\lambda-4}{\mu+4}
\end{gathered}
$$

Finally, substituting $\lambda=\mu=0$, we have the desired expected values :

$$
\begin{aligned}
u_{3}\left(s_{1} ; 0,0\right) & =\frac{1}{4^{3}} \cdot \frac{8}{16}+\frac{3 \cdot 5}{4^{3}} \cdot \frac{5}{13}+\frac{3 \cdot 6}{2 \cdot 4^{2}} \cdot \frac{1}{5}+\frac{3}{2^{2} \cdot 4} \cdot\left(-\frac{1}{7}\right) \\
& =\cdots=\frac{9969}{58240}=0.17117101648 \\
u_{3}\left(s_{2} ; 0,0\right) & =\frac{1}{2 \cdot 4^{2}} \cdot \frac{5}{13}+\frac{11}{2 \cdot 4^{2}} \cdot \frac{1}{5}+\frac{1}{2} \cdot\left(\frac{-1}{7}\right)+\frac{1}{2^{3}} \cdot(-1) \\
& =\cdots=-\frac{421}{25480}=-0.01652276295
\end{aligned}
$$




\section{Concluding Remarks}

We have considered the conditional expected value of the related associative statistics $R_{0} \circ R_{1} \circ \cdots \circ R_{n}$. Firstly we treat the conditional expected value of $R_{1} \circ R_{2} \circ \cdots \circ R_{n}$ instead:

$$
u_{n}(i)=E\left[R_{1} \circ R_{2} \circ \cdots \circ R_{n} \mid X_{0}=i\right] \quad i \in S
$$

Let

$$
u_{n}(i ; \lambda)=E\left[\lambda \circ R_{1} \circ R_{2} \circ \cdots \circ R_{n} \mid X_{0}=i\right] \quad \lambda \in R^{1} .
$$

Then, we get the forward recurrence equation:

$$
\begin{aligned}
& u_{1}(i ; \lambda)=\sum_{j=1}^{N}(\lambda \circ r(j)) p(j \mid i) \\
& u_{n}(i ; \lambda)=\sum_{j=1}^{N} u_{n-1}(j ; \lambda \circ r(j)) p(j \mid i) \quad n \geq 2 .
\end{aligned}
$$

On the other hand, we imbed the problem (16) into the class of backward problems: $u^{n-k}(i ; \lambda)=E\left[\lambda \circ R_{k+1} \circ R_{k+2} \circ \cdots \circ R_{n} \mid X_{k}=i\right] \quad 0 \leq k \leq n-1, \quad \lambda \in R^{1}$.

We have the backward recurrence equation:

$$
\begin{aligned}
u^{1}(i ; \lambda) & =\sum_{j=1}^{N}(\lambda \circ r(j)) p(j \mid i) \\
u^{n-k}(i ; \lambda) & =\sum_{j=1}^{N} u^{n-k-1}(j ; \lambda \circ r(j)) p(j \mid i) \quad 0 \leq k \leq n-2 .
\end{aligned}
$$

Secondly we consider the expected values of the following nonassociative statistics

$$
u_{n}(i)=E\left[\left(\left(\cdots\left(\left(R_{0} \circ R_{1}\right) \circ R_{2}\right) \circ \cdots\right) \circ R_{n}\right) \mid X_{0}=i\right] \quad i \in S
$$

where the nonassociativity does not assure the equality

$$
(x \circ y) \circ z=x \circ(y \circ z) .
$$

We also imbed the problem (17) into the class of forward problems:

$$
u_{n}(i ; \lambda)=E\left[\left(\left(\cdots\left(\left(\lambda \circ R_{0}\right) \circ R_{1}\right) \circ \cdots\right) \circ R_{n}\right) \mid X_{0}=i\right] \quad \lambda \in R^{1}
$$

Then we have the forward recurrence equation:

$$
\begin{aligned}
& u_{0}(i ; \lambda)=\lambda \circ r(i) \\
& u_{n}(i ; \lambda)=\sum_{j=1}^{N} u_{n-1}(j ; \lambda \circ r(i)) p(j \mid i) \quad n \geq 1
\end{aligned}
$$


This system has the same form as the system (4),(5). Solving the system, we can calculate the expected value of forward exponential statistics :

$$
u_{n}(i)=E\left[R_{n}^{R_{n-1}^{\cdots R_{1}^{R_{0}}}} \mid X_{0}=i\right] \quad i \in S .
$$

Moreover, we can obtain the corresponding (nonstationary) recurrence equation for class of forward problems with nonstationary binary relations:

$$
u_{n}(i ; \lambda)=E\left[\left(\left(\cdots\left(\left(\lambda * R_{0}\right) \diamond R_{1}\right) \circ \cdots\right) \bullet R_{n}\right) \mid X_{0}=i\right] \quad \lambda \in R^{1},
$$

where $*, \diamond, \circ, \cdots, \bullet$ are binary relations. For instance, the problem

$$
\left.u_{3}(i)=E\left[\left(\left(R_{0} \wedge R_{1}\right) R_{2}\right)+R_{3}\right)^{R_{4}} \mid X_{0}=i\right]
$$

belongs to the class. Because we have

$$
u_{3}(i)=E\left[\left(\left(\left(R_{0} * R_{1}\right) \diamond R_{2}\right) \circ R_{3}\right) \bullet R_{4} \mid X_{0}=i\right]
$$

where

$$
a * b=a \wedge b, a \diamond b=a b, \quad a \circ b=a+b, a \bullet b=a^{b} .
$$

Finally we remark that we have also the resulting backward equation (7),(8) for the nonassociative statistics (17).

\section{References}

Bellman, R.E. (1957), Dynamic Programming, Princeton Univ. Press, NJ.

Bellman, R.E. and Denman, E.D. (1971), Invariant Imbedding, Lect. Notes in Operation Research and Mathematical Systems, Vol. 52, Springer-Verlag, Berlin.

Bellman, R.E. and Zadeh, L.A. (1970), Decision-making in a fuzzy environment, Management Sci., 17:B141-B164.

Blackwell, D. (1965), Discounted dynamic programming, Ann. Math. Stat., 36:226235.

Denardo, E.V. (1968), Contraction mappings in the theory underlying dynamic programming, SIAM Review, 9:165-177.

Denardo, E.V. (1982), Markov Dynamic Programming: Models and Applications, Prentice-Hall, N.J.

Esogbue, A.O. and Bellman, R.E. (1984), Fuzzy dynamic programming and its extensions, TIMS/Studies in the Management Sciences, 20:147-167.

Furukawa, N. and Iwamoto, S. (1973), Markovian decision processes with recursive reward functions, Bull. Math. Statist., 15:79-91.

Furukawa, N. and Iwamoto, S. (1973), Dynamic programming on recursive reward systems, Bull. Math. Statist., 17:103-126.

Golmb,S.W. (1968), E2118, Amer. Math. Monthly, 75:878. 
Golmb,S.W. (1980), Iterated binomial coefficients, Amer. Math. Monthly, 87:719-727.

Hinderer, K. (1970), Foundations of Non-Stationary Dynamic Programming with Discrete Time Parameter, Lect. Notes in Operation Research and Mathematical Systems, Vol. 33, Springer-Verlag, Berlin.

Howard, R.A. (1960), Dynamic Programming and Markov Processes, MIT Press, Cambridge, Mass.

Iwamoto, S. (1974), Discrete dynamic programming with recursive additive system, Bull. Math. Statist., 15:49-66.

Iwamoto, S. (1975a), Linear programming on recursive additive dynamic programming, Bull. Math. Statist., 18:125-151.

Iwamoto, S. (1975b), Finite-horizon Markov games with recursive payoff systems, Mem. Fac. Sci. Kyushu Univ. Ser. A, 29:123-147.

Iwamoto, S. (1977), The second principle of optimality, Bull. Math. Statist., 17:104114.

Iwamoto, S. (1993), From dynamic programming to bynamic programming, J. Math. Anal. Appl., 177:56-74.

Iwamoto, S. (1994), On bidecision processes, J. Math. Anal. Appl., 187:676-699.

Iwamoto, S. (1996), Associative dynamic programs, J. Math. Anal. Appl., 201:195211.

Iwamoto, S. and Fujita, T. (1995), Stochastic decision-making in a fuzzy environment, J. Operations Res. Soc. Japan, 38:467-482.

Kacprzyk, J. (1978), Decision-making in a fuzzy environment with fuzzy termination time, Fuzzy Sets and Systems, 1:169-179.

Kreps, D.M. (1977a), Decision problems with expected utility criteria, I, Math. Oper. Res., 2:45-53.

Kreps, D.M. (1977b), Decision problems with expected utility criteria, II, Math. Oper. Res., 2:266-274.

Lee, E.S. (1968), Quasilinearization and Invariant Imbedding, Academic Press, New York.

Lipfert, W. (1985), Über ein stochastisches dynamiches Entscheidungsmodell mit allegemeinen Ertragsfunktionalen, Optimization, 16:313-328.

Mitten, L.G. (1964), Composition principles for sysnthesis of optimal multi-stage processes, Operations Res., 12:610-619.

Nemhauser, G.L. (1966), Introduction to Dynamic Programming, Wiley, New York.

Porteus, E. (1975), An informal look at the princiole of optimality, Management Sci., $21: 1346-1348$.

Porteus, E. (1982), Conditions for characterizing the structure of optimal strategies in infinite-horizon dynamic programs, J. Opt. Theo. Anal, 36:419-432.

Puterman, M.L. (1994), Markov Decision Processes : discrete stochastic dynamic programming, Wiley \& Sons, New York.

Sniedovich, M. (1986), A new look at Bellman's Principle of Optimality, J. Opt. Theo. Anal., 49:161-176. 
Sniedovich, M. (1987), A class of nonseparable dynamic programming problems, $J$. Opt. Theo. Anal., 52:111-121.

Sniedovich, M. (1983), A class of variance constrained problems, Operations Research, 31:338-353.

Sniedovich, M. (1989), Analysis of a class of fractional programming problems, Math. Prog., 43:329-347.

Sniedovich, M. (1992), Dynamic Programming, Marcel Dekker, Inc. NY.

Received June 27, 1997 